\title{
On metamaterial circular polarizers based on metal $N$-helices
}

\author{
Johannes Kaschke, ${ }^{1,2, *}$ Justyna K. Gansel, ${ }^{1}$ and Martin Wegener ${ }^{1,2}$ \\ ${ }^{1}$ Institute of Applied Physics and DFG-Center for Functional Nanostructures (CFN), Karlsruhe Institute of \\ Technology (KIT), 76131 Karlsruhe, Germany \\ ${ }^{2}$ Institute of Nanotechnology (INT), Karlsruhe Institute of Technology (KIT), 76131 Karlsruhe, Germany \\ "Johannes.Kaschke@kit.edu
}

\begin{abstract}
Metal-helix based metamaterials have been introduced as compact and broadband circular polarizers. However, the end of the metal wire together with the helix center defines an axis in space, which unavoidably breaks the rotational symmetry at the metamaterial surface. This introduces linear birefringence. Symmetry can be recovered by considering an integer number, e.g. $N=4$, of intertwined helices arranged to a square array. We show that the operation principles are fundamentally different though. Metamaterial circular polarizers based on $N=4$ helices, unlike single helices, inherently require absorption of the constituent metal. Otherwise, the combination of a four-fold rotational axis and time-inversion symmetry strictly forbids circular-polarizer action. Our symmetry analysis is confirmed by extensive numerical calculations comparing results for perfect electric conductors with those for a free-electron Drude metal with finite damping.
\end{abstract}

(C)2012 Optical Society of America

OCIS codes: (160.1585) Materials: Chiral media; (160.3918) Materials: Metamaterials; (260.5430) Physical optics: Polarization.

\section{References and links}

1. J. K. Gansel, M. Thiel, M. S. Rill, M. Decker, K. Bade, V. Saile, G. von Freymann, S. Linden, and M. Wegener, "Gold helix photonic metamaterial as broadband circular polarizer," Science 325(5947), 1513-1515 (2009).

2. J. K. Gansel, M. Wegener, S. Burger, and S. Linden, "Gold helix photonic metamaterials: A numerical parameter study," Opt. Express 18(2), 1059-1069 (2010).

3. C. Wu, H. Li, Z. Wei, X. Yu, and C. T. Chan, "Theory and experimental realization of negative refraction in a metallic helix array," Phys. Rev. Lett. 105(24), 247401 (2010).

4. M. Thiel, M. S. Rill, G. von Freymann, and M. Wegener, "Three-dimensional bichiral photonic crystals," Adv. Mater. 21, 4680-4682 (2009).

5. A. Radke, T. Gissibl, T. Klotzbücher, P. V. Braun, and H. Giessen, "Three-dimensional bichiral plasmonic crystals fabricated by direct laser writing and electroless silver plating," Adv. Mater. (Deerfield Beach Fla.) 23(27), 3018-3021 (2011).

6. J. K. Gansel, M. Latzel, A. Frölich, J. Kaschke, M. Thiel, and M. Wegener, "Tapered gold-helix metamaterials as improved circular polarizers," Appl. Phys. Lett. 100(10), 101109 (2012).

7. M. Thiel, G. von Freymann, and M. Wegener, "Layer-by-layer three-dimensional chiral photonic crystals," Opt. Lett. 32(17), 2547-2549 (2007).

8. M. Decker, R. Zhao, C. M. Soukoulis, S. Linden, and M. Wegener, "Twisted split-ring-resonator photonic metamaterial with huge optical activity," Opt. Lett. 35(10), 1593-1595 (2010).

9. Y. Zhao, M. A. Belkin, and A. Alù, "Twisted optical metamaterials for planarized ultrathin broadband circular polarizers," Nat Commun 3, 870 (2012).

10. Z. Y. Yang, M. Zhao, P. X. Lu, and Y. F. Lu, "Ultrabroadband optical circular polarizers consisting of doublehelical nanowire structures," Opt. Lett. 35(15), 2588-2590 (2010).

11. Z. Yang, M. Zhao, and P. Lu, "Improving the signal-to-noise ratio for circular polarizers consisting of helical metamaterials," Opt. Express 19(5), 4255-4260 (2011).

12. J. D. Kraus and R. Marhefka, Antennas: For All Applications, 3rd ed. (McGraw-Hill, 2003).

13. R. J. Potton, "Reciprocity in optics," Rep. Prog. Phys. 67(5), 717-754 (2004).

14. J. D. Jackson, Classical Electrodynamics, 3rd ed. (John Wiley \& Sons, 1999).

15. E. Hecht, Optics, 4th ed. (Addison-Wesley, 2002). 
16. I. V. Lindell, A. H. Sihvola, S. A. Tretyakov, and A. J. Viitanen, Electromagnetic Waves in Chiral and BiIsotropic Media (Artech House, 1994).

17. C. E. Kriegler, M. S. Rill, S. Linden, and M. Wegener, "Bianisotropic photonic metamaterials," IEEE J. Sel. Top. Quantum Electron. 16(2), 367-375 (2010).

18. J. Fischer and M. Wegener, "Three-dimensional optical laser lithography beyond the diffraction limit," Laser Photon. Rev. doi: 10.1002/lpor.201100046.

\section{Introduction}

Gold-helix metamaterials have been introduced as compact and broadband circular polarizers [1-3]. They can be seen as the circular counterpart of the good old wire-grid polarizer for linear polarization of light. Simple square arrays of circular helices with two and more pitches along the helix axis provide an operation bandwidth of nearly one octave [1] (for light propagating along the helix axis). Within this bandwidth, one circular polarization is transmitted, the other one is reflected. Metal absorption is not required and is actually rather unwanted. Several variations of this idea have meanwhile been published aiming at further improving the circular-polarizer performance. For example, three-dimensional bi-chiral arrangements [4,5] promise a more isotropic response, however, they largely deteriorate both the bandwidth and the extinction ratio [5]. Tapered helices increase the bandwidth to about 1.5 octaves, increase the extinction, and reduce polarization conversion [6]. Simplified layerby-layer chiral designs [7-9] cannot quite match the performance of helix-based metamaterials, but these structures are closer to practical applications and allow for much larger sample footprints due to an inherently simpler fabrication process [9].

When looking at a circular helix along the helix axis (see Fig. 1(a)), the end of the wire forming the helix together with the central axis of the helix form a direction in space, which breaks the wanted full rotational symmetry of the chiral metamaterial [2]. In transmission, this fact leads to a small but unwanted degree of circular polarization conversion, e.g., incident left-handed circular polarization (LCP) is partially converted to emerging right-handed circular polarization (RCP). In reflection, this conversion effect is dramatic [2]. For a bulk metal, a bulk dielectric, or for an ideal isotropic chiral effective material under normal incidence of light onto a sample surface, e.g., LCP would be reflected as pure RCP. In sharp contrast, for metal-helix metamaterials, incident LCP is reflected as LCP, i.e., one gets nearly $100 \%$ of polarization conversion in reflection [2].

To reduce or eliminate these unwanted polarization conversion effects, multiple (integer $N$ ) intertwined helices have previously been proposed [10,11]. For $N=2$, they can be seen as the analog to the DNA double strands in biology. At first sight, one might be tempted to look at an $\mathrm{N}$-helix metamaterial as a small modification of a metamaterial composed of single helices. In this work, we show that the operation principles are fundamentally different due to different symmetry. As a result, the optimization of the circular-polarizer action is very different, too.

\section{Symmetry based analysis}

Figure 1 illustrates unit cells of simple square arrays of single helices and multiple helices for $N=4$. In the $N=4$ case in (b), the symmetry of a single building block ("meta-atom") and that of the array are identical. Both exhibit a four-fold rotational symmetry. $N=1,2,3, \ldots$ do not fulfill this condition, $N=8,12,16, \ldots$ would. However, the difficulty of fabrication obviously rapidly increases with increasing $N$. Thus, we only explicitly consider $N=4$ in this letter. It is known that the operation bandwidth depends on the number of helix pitches along the helix axis [1,2]. We start our discussion with single pitches, where the resonances are simpler to understand [1]. At the end of this paper, we also consider several axial pitches for $N=4$ helices. The dependence of the optical properties of single helices on the number of pitches resembles the expectation from end-fire helical antenna theory [12], has previously been published $[1,2]$, and shall not be repeated here.

\#174093 - \$15.00 USD Received 9 Aug 2012; revised 27 Sep 2012; accepted 28 Sep 2012; published 2 Nov 2012

(C) 2012 OSA

5 November 2012 / Vol. 20, No. 23 / OPTICS EXPRESS 26013 


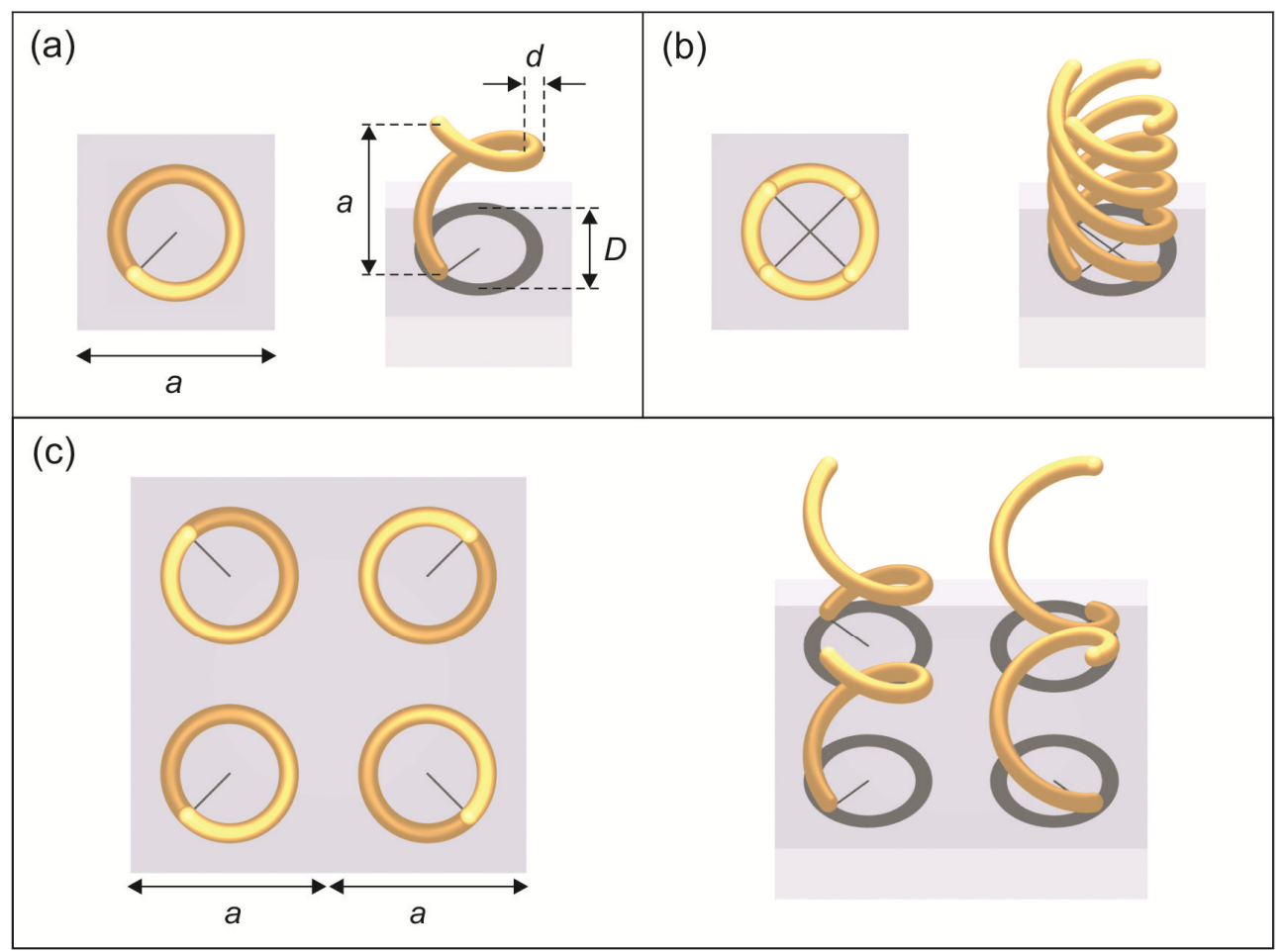

Fig. 1. Illustration of the unit cells of helix-metamaterial geometries considered in this work. In each case, top view (left) and oblique view (right) are depicted. (a) Single $(N=1)$ left-handed metal helices with axial period $a$ arranged to a square array with lateral lattice constant $a$. The metal wire diameter is $d=0.1 \times a$, the helix diameter is $D=0.6 \times a$. Note that the end of the metal wire together with the center axis of the helices defines an axis in space. This leads to a one-fold rotational axis. (b) Similarly arranged $N=4$ intertwined helices recover a four-fold rotational axis compatible with the square-array symmetry. (c) Arrangement of single helices like in (a) effectively recovering four-fold rotational symmetry of the overall structure by laterally displacing the four helices from (b) within one new unit cell with lattice constant $2 a$.

For single helices, we have previously shown [1] that the circular-polarizer effect survives if the metal is described as a perfect electric conductor (PEC). For a PEC, absorption is strictly zero by definition. Thus, any difference in the metamaterial transmittance for incident LCP and RCP must be due to a difference in reflectance at the interfaces. For the case of $N=$ 4 helices we now show that, due to symmetry, the intensity reflectance and transmittance coefficients for LCP and RCP are strictly identical.

Importantly, we shall not use the effective-medium approximation, the validity of which is somewhat questionable under our conditions, but rather consider the sample as a "black box" and only exploit its symmetries. Consider a plane wave of light impinging along the axis of the helices, i.e., along the $z$-axis. Thus, the electric field has components in the $x y$-plane. The reflected field vector $\vec{E}_{\mathrm{r}}$ can be connected to the incident field vector $\vec{E}_{\mathrm{i}}$ by the fieldreflection 2x2 matrix $\vec{r}$ with complex-valued coefficients given by

$$
\vec{E}_{\mathrm{r}}=\vec{r} \vec{E}_{\mathrm{i}}=\left(\begin{array}{cc}
r_{x x} & r_{x y} \\
r_{y x} & r_{y y}
\end{array}\right) \vec{E}_{\mathrm{i}}
$$

Here, we have obviously tacitly neglected any diffracted orders. This assumption is justified for the conditions of interest in this work. For a four-fold symmetry of the structure, $\vec{r}$ must be invariant under rotations of 90 degrees and multiples thereof, i.e., $\vec{r}$ must commute with 
the 90-degree rotation matrix $\vec{M}_{90}$. This is equivalent to invariance under the coordinate transformation $\vec{r}=\vec{M}_{90} \vec{r} \vec{M}_{90}^{-1}$. This condition leads to $r_{x x}=r_{y y}$ and $r_{y x}=-r_{x y}$. Thus we have

$$
\vec{r}=\left(\begin{array}{cc}
r_{x x} & r_{x y} \\
-r_{x y} & r_{x x}
\end{array}\right)
$$

To make the transition from this linear-polarization basis to a circular-polarization basis, we use the usual matrix $\vec{S}$ given by

$$
\vec{S}=\frac{1}{\sqrt{2}}\left(\begin{array}{cc}
1 & -\mathrm{i} \\
1 & \mathrm{i}
\end{array}\right)
$$

The reflection coefficients in circular ("circ") basis become

$$
\vec{r}_{\text {circ }}=\vec{S} \vec{r} \vec{S}^{-1}=\left(\begin{array}{cc}
r_{x x}+\mathrm{i} r_{x y} & 0 \\
0 & r_{x x}-\mathrm{i} r_{x y}
\end{array}\right)=\left(\begin{array}{cc}
r_{\mathrm{RCP} \mathrm{LCP}} & 0 \\
0 & r_{\mathrm{LCP} \mathrm{RCP}}
\end{array}\right) .
$$

As argued above, incident LCP is reflected as RCP and vice versa. As usual, this change is simply due to the fact that the sense of rotation of the electric-field vector stays the same but the axis it is referred to, namely the wave vector of light, changes direction upon reflection. The zero off-diagonal elements in Eq. (4) mean that circular polarization conversion is strictly zero. By analogous reasoning, we can derive a transmission matrix $\vec{t}_{\text {circ }}$ with nonzero diagonal elements $t_{\mathrm{LCP} L \mathrm{LP}}$ and $t_{\mathrm{RCP} \text { RCP }}$ and zero off-diagonal elements.

Next, we exploit time-inversion symmetry. Consider an incident field vector in the circular basis, $\vec{E}_{\mathrm{i}, \text { circ }}$, with components $E_{\mathrm{i}, \mathrm{LCP}}$, and $E_{\mathrm{i}, \mathrm{RCP}}$. As the conversions are zero, we can, for example, consider solely $E_{\mathrm{i}, \mathrm{LCP}}$. This leads to the transmitted and reflected field vector components

$$
E_{\mathrm{t}, \mathrm{LCP}}=t_{\mathrm{LCP} L C P} E_{\mathrm{i}, \mathrm{LCP}} \quad \text { and } \quad E_{\mathrm{r}, \mathrm{RCP}}=t_{\mathrm{RCP} \mathrm{LCP}} E_{\mathrm{i}, \mathrm{LCP}} .
$$

Upon time inversion, not only the time $t \rightarrow-t$, but also the wave vector $\vec{k} \rightarrow-\vec{k}$ and any relative phase between the two vector components have to be inverted. Hence, the field vectors turn into their complex conjugates, see, e.g [13-15]. We now consider $E_{\mathrm{t}, \mathrm{LCP}}^{*}$ and $E_{\mathrm{r}, \mathrm{RCP}}^{*}$ as the incident field vector components. In general, the structure must not necessarily be symmetric with respect to inverting the propagation direction. For the incident field $E_{\mathrm{t}, \mathrm{LCP}}^{*}$, that is propagating in the opposite direction we therefore introduce the reflection and transmission matrices $\vec{r}_{\text {circ }}^{\prime}$ and $\vec{t}_{\text {circ }}^{\prime}$. Both matrices will again have zero off-diagonal elements due to four-fold rotational symmetry. Due to time-inversion symmetry [15], the following two equations must be satisfied

$$
\begin{aligned}
& 0=t_{\mathrm{RCP} \mathrm{RCP}} \mathrm{r}_{\mathrm{RCP} \mathrm{LCP}}^{*}+r_{\mathrm{RCP} \mathrm{LCP}}^{\prime} t_{\mathrm{LCP} \mathrm{LCP}}^{*} \\
& 1=r_{\mathrm{LCP} \mathrm{RCP}} \mathrm{r}_{\mathrm{RCP} \mathrm{LCP}}^{*}+t_{\mathrm{LCP} \mathrm{LCP}}^{\prime} t_{\mathrm{LCP} \mathrm{LCP}}^{*} .
\end{aligned}
$$

\#174093 - \$15.00 USD

(C) 2012 OSA 
As the structure is assumed to be lossless, electromagnetic energy must be conserved. This yields

$$
1=r_{\mathrm{RCPLCP}}^{\prime} \mathrm{r}_{\mathrm{RCP} L C P}^{*}+t_{\mathrm{LCP} \mathrm{LCP}}^{\prime} t_{\mathrm{LCP} L \mathrm{LP}}^{*}
$$

By solving Eqs. (6) and (7) for $r_{\text {RCPLCP }}^{\prime}$ and $t_{\text {LCP LCP }}^{\prime}$ respectively and substituting appropriately into Eq. (8) we get

$$
\left|t_{\mathrm{LCPLCP}}\right|^{2}-\left|t_{\mathrm{RCP} \mathrm{RCP}}\right|^{2}\left|r_{\mathrm{RCPLCP}}\right|^{2}=\left|1-r_{\mathrm{LCP} \mathrm{RCP}} r_{\mathrm{RCPLCP}}^{*}\right|^{2} \text {. }
$$

A similar equation can be derived for incident RCP

$$
\left|t_{\mathrm{RCP} \mathrm{RCP}}\right|^{2}-\left|t_{\mathrm{LCP} \mathrm{LCP}}\right|^{2}\left|r_{\mathrm{LCP} \mathrm{RCP}}\right|^{2}=\left|1-r_{\mathrm{RCP} \mathrm{LCP}} r_{\mathrm{LCP} \mathrm{RCP}}^{*}\right|^{2} .
$$

The squared moduli on the right-hand side of Eqs. (9) and (10) are equal and we therefore have

$$
\left|t_{\mathrm{LCPLCP}}\right|^{2}-\left|t_{\mathrm{RCP} \mathrm{RCP}}\right|^{2}\left|r_{\mathrm{RCP} \mathrm{LCP}}\right|^{2}=\left|t_{\mathrm{RCP} \mathrm{RCP}}\right|^{2}-\left|t_{\mathrm{LCPLCP}}\right|^{2}\left|r_{\mathrm{LCPRCP}}\right|^{2}
$$

Exploiting conservation of electromagnetic energy, i.e., $\quad 1=\left|r_{\mathrm{RCPLCP}}\right|^{2}+\left|t_{\mathrm{LCPLCP}}\right|^{2}$ and $1=\left|r_{\mathrm{LCPRCP}}\right|^{2}+\left|t_{\mathrm{RCPRCP}}\right|^{2}$, this finally yields

$$
\left|t_{\mathrm{LCPLCP}}\right|=\left|t_{\mathrm{RCP} \text { RCP }}\right|
$$

and accordingly

$$
\left|r_{\mathrm{RCP} \mathrm{LCP}}\right|=\left|r_{\mathrm{LCP} \mathrm{RCP}}\right| \text {. }
$$

We note that this finding is consistent with and well known for bi-isotropic effective media [16], a subclass of bi-anisotropic effective media [17]. However, as the wavelength is not really much larger than the characteristic feature sizes for the helix structures of interest here, we feel that a reasoning based on the effective-medium approximation would not have been "water-proof".

Altogether, lossless $N=4$ helices exhibit

1. four-fold rotational symmetry along the helix axis and

2. time-inversion symmetry (no losses and no static magnetic field).

We consider the case that no diffracted orders other than the 0th orders in the forward and backward directions emerge. In this case, symmetry 1. alone implies that the circular polarization conversion is strictly zero for transmission and reflection. This aspect is desired. Unfortunately, the combination of symmetries 1. and 2. also implies that the intensity reflection coefficients for LCP and RCP are strictly identical (at any frequency). The same holds true for the LCP and RCP intensity transmission coefficients. The field transmission coefficients for LCP and RCP can have different phases though (like in, e.g., an optically active solution of chiral sugar molecules). This overall finding is quite counter-intuitive: The $N=4$ helix structure is chiral - but its optical properties show hardly any traces of that. In particular, the structure just cannot act as a circular polarizer.

However, finite losses would break time-inversion symmetry. In this case, different transmissions may result from finite propagation losses that can be different for LCP and RCP respectively due to the chiral nature of the helices. 


\section{Numerical calculations}

To test our symmetry considerations, we compare them to numerical calculations using the commercial software package CST Microwave Studio based on a finite-integration technique. The structure parameters for $N=1$ and $N=4$ are illustrated in Fig. 1(a) and 1(b) respectively. We choose $a=1 \mu \mathrm{m}$. We have performed calculations with and without the glass substrate, which does break the symmetry with respect to inverting the propagation direction [6]. The differences turn out to be very small though. Thus, for clarity, we only depict results without glass substrate here. For the frequencies depicted in Figs. 2 and 3, diffraction into free space cannot occur (the high-frequency end of about $300 \mathrm{THz}$ corresponds to a free-space wavelength of $1 \mu \mathrm{m}=a$ ).

We only show results for left-handed helices. For right-handed helices, LCP just needs to be exchanged by RCP and vice versa (by symmetry).

We start our discussion with the conceptually simple case of metal helices made of a PEC, for which losses are strictly zero. It becomes obvious from Fig. 2(a) that the single-helix metamaterial does act as a circular polarizer, whereas the $N=4$ helix metamaterial in Fig. 2(b) shows strictly zero difference in intensity reflectance and transmittance between LCP and $\mathrm{RCP}$ at any frequency. This highlights the fundamental difference between the $N=1$ and the $N=4$ case, in agreement with our symmetry considerations in the preceding section. Energy conservation for a PEC means that the sum of all red curves in Fig. 2(a) at a given frequency is one (same for Fig. 2(b)). The same holds true for the blue curves. Our numerical calculations obey energy conservation to better than $1 \%$. Within the PEC limit, due to the scalability of the Maxwell equations, the operation frequency can simply be scaled to any desired frequency.

However, for small structures and high operation frequencies, the PEC assumption becomes unrealistic and the metal rather has to be described by the Drude free-electron model, which does lead to finite losses. Corresponding calculations for gold with plasma frequency $\omega_{\mathrm{PL}}=1.37 \times 10^{16} \mathrm{rad} / \mathrm{s}$ and collision frequency $\omega_{\text {col }}=1.2 \times 10^{14} \mathrm{rad} / \mathrm{s}$ are shown in Fig. 2(c) and (d), where again $a=1 \mu \mathrm{m}$. When going from PEC to gold, the performance of the single-helix metamaterial in (c) deteriorates with respect to (a). In sharp contrast, the PEC $N=4$ helix metamaterial in Fig. 2(b) shows no circular-polarizer action at all, while at least some difference between LCP and RCP intensity transmittance is found in (d). This highlights once again the fundamental difference in operation principles between the $N=1$ and the $N=4$ case. In agreement with our symmetry considerations in the preceding section, all polarization conversions (dashed curves) are strictly zero for the $N=4$ case, with and without losses.

So far, we have only discussed metamaterials composed of single helix pitches in this paper. The $N=1$ case is known [1] to exhibit significantly larger bandwidth for two or more pitches along the helix axis, which is identical to the propagation direction of light. Figure 3 shows that the behavior is similar for the $N=4$ helix metamaterial. When going from Fig. $3(a)$ to $3(d)$, the extinction depth increases with increasing number of pitches. This is simply due to the fact that one circular polarization (LCP) experiences much larger resonant absorption losses than the other one (RCP). Thus, with increasing number of pitches, hence increasing sample thickness, the difference in optical density between LCP and RCP increases. At the same time, the operation bandwidth (see gray areas) increases from one to six pitches. Intuitively, the bandwidth larger than a factor of three (i.e., 1.5 octaves) stems from the strong interaction among the different helix pitches [1], somewhat similar to the interaction of the primary and the secondary coil in a transformer [2]. At the same time, the operation bandwidth (see gray areas) increases from one to six pitches. Intuitively, the bandwidth larger than a factor of three (i.e., 1.5 octaves) stems from the strong interaction among the different helix pitches [1], somewhat similar to the interaction of the primary and the secondary coil in a transformer [2]. 

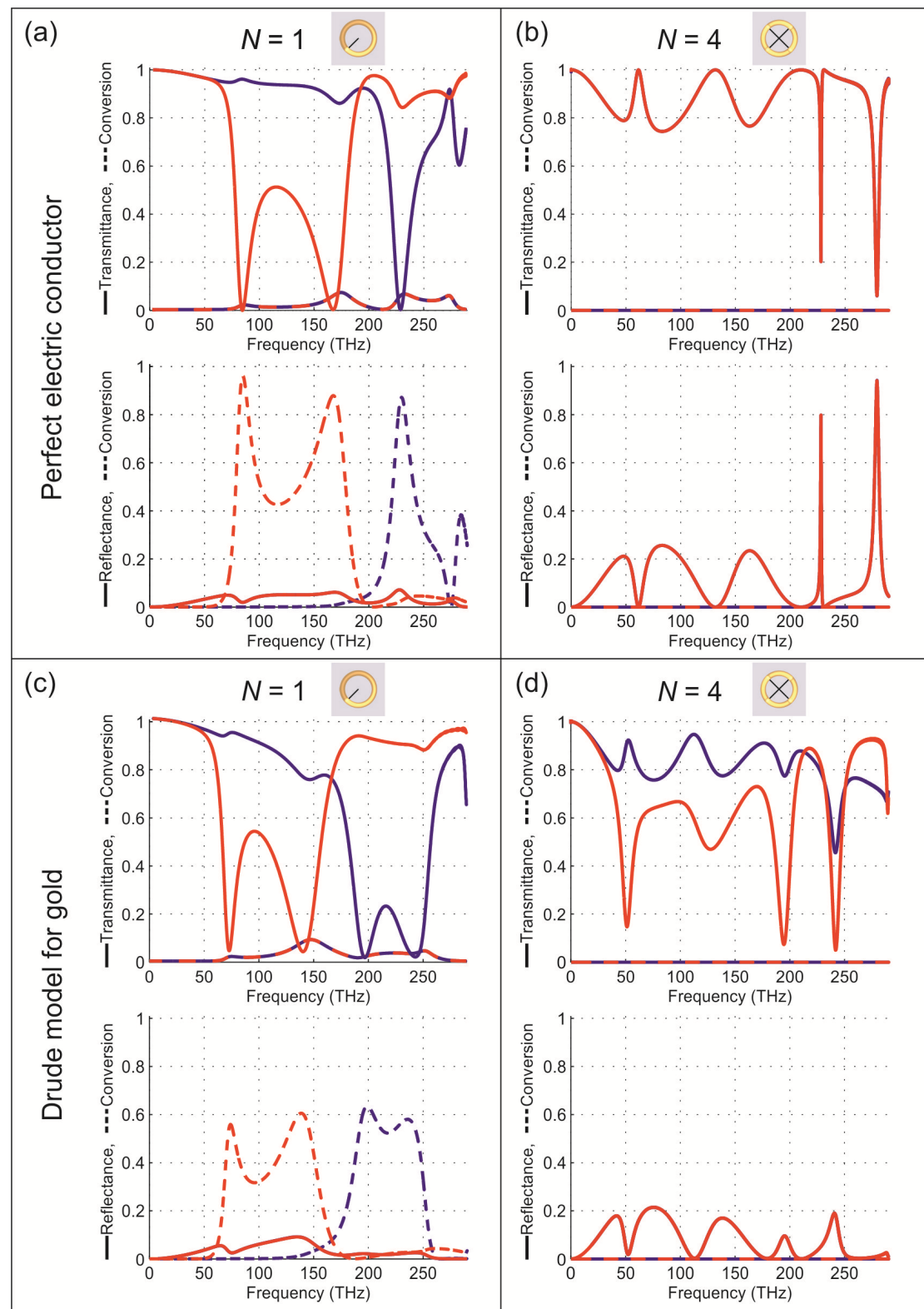

Fig. 2. Calculated normal-incidence intensity transmittance (solid), reflectance (solid), and conversion spectra (dashed). Incident left-handed circular polarization (LCP) is shown in red, incident right-handed circular polarization (RCP) in blue. In cases where no blue curve is visible, the blue curve is identical to the red one to within the curve linewidth. The left-handed $N=1$ (left column, i.e., (a) and (c)) and $N=4$ (right column, i.e., (b) and (d)) structures are defined in Fig. 1(a) and Fig. 1(b), respectively. The insets at the top repeat the top view onto a unit cell. For the top row of the overall $2 \times 2$ matrix (i.e., (a) and (b)), the metal is treated as a lossless perfect electric conductor (PEC), for the bottom row (i.e., (c) and (d)) as a freeelectron Drude model with finite damping/losses (gold parameters). 


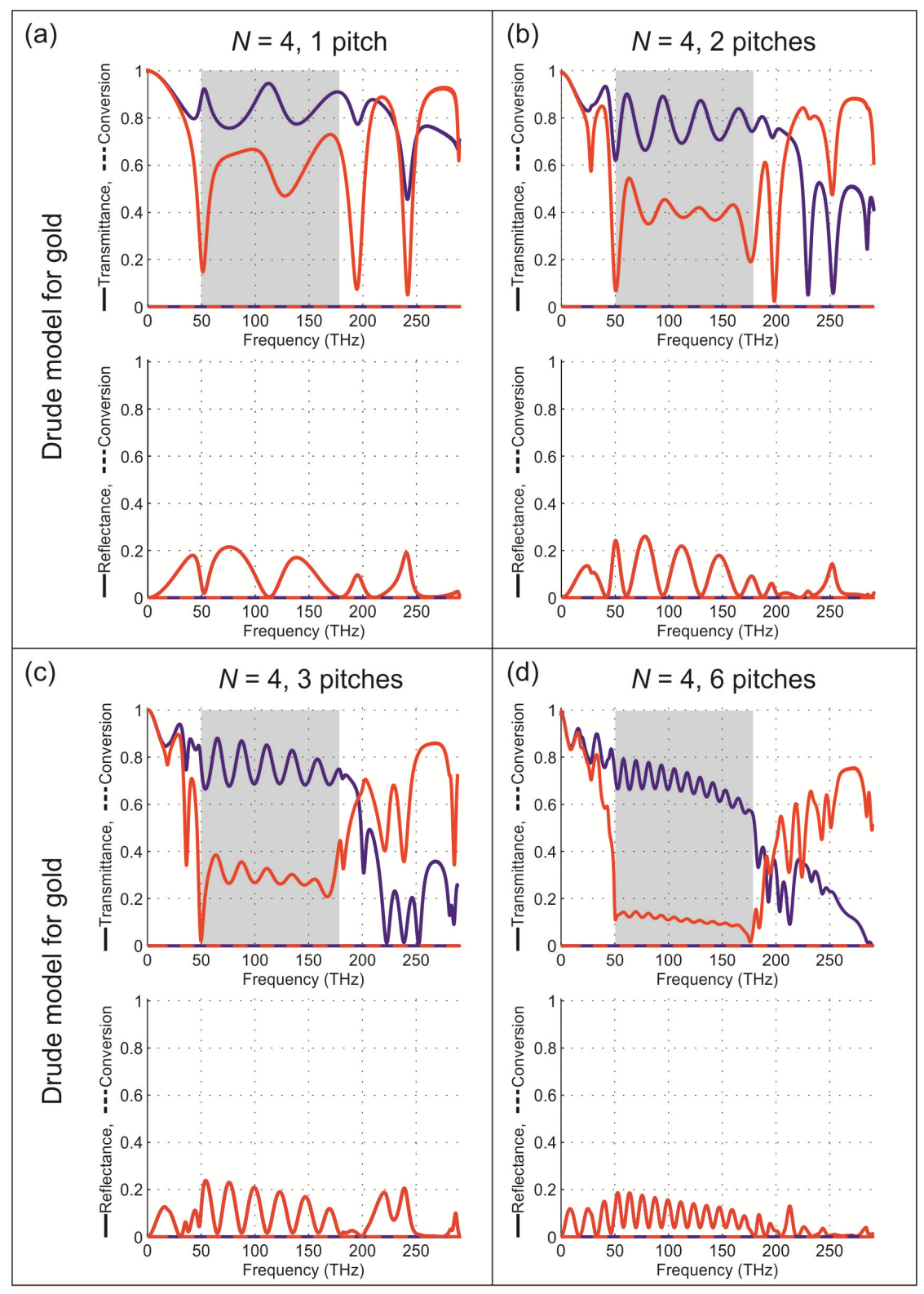

Fig. 3. As Fig. 2(d), but for more than one helix pitch along the helix axis as indicated. (a) same as Fig. 2(d) with 1 pitch, (b) 2 pitches, (c) 3 pitches, and (d) 6 pitches. Note that all conversions (dashed) are strictly zero. In reflection, no blue solid curves are visible because they are identical to the red ones to within the curve linewidth. The equal gray areas in (a)-(d) highlight the frequency interval for which broadband circular-polarizer action is observed for several axial pitches.

Finally, we briefly mention that our above symmetry reasoning also applies to the metamaterial structure illustrated in Fig. 1(c). Here, four-fold rotational symmetry is recovered by changing the rotation of the adjacent $N=1$ helices in one larger unit cell. Our corresponding numerical calculations (not depicted) exhibit zero circular polarization conversion for transmittance and reflectance and strictly no circular-polarizer action for any frequency within the PEC limit, just like for the $N=4$ helices in Fig. 2(b).

\#174093 - \$15.00 USD Received 9 Aug 2012; revised 27 Sep 2012; accepted 28 Sep 2012; published 2 Nov 2012

(C) 2012 OSA

5 November 2012 / Vol. 20, No. 23 / OPTICS EXPRESS 26019 


\section{Conclusions}

In conclusion, due to four-fold rotational symmetry, the optical properties of metamaterials based on square arrays of $N=4$ helices are fundamentally different from those of metamaterials based on $N=1$ helices. For the $N=4$ case, one does completely eliminate circular polarization conversion for both reflection and transmission, whereas polarization conversion is inherently unavoidable for $N=1$. Unfortunately, without metal losses, one also completely eliminates the desired circular-polarizer effect for $N=4$. The circular-polarizer effect can be recovered for finite metal losses. This implies that the structure is no longer easily scalable to any operation frequency and that certain inherent losses must be accepted. On the positive side, circular polarization conversion is strictly zero for $N=4$ in reflection and transmission even in the presence of losses.

Normal direct laser writing (DLW) has allowed for realizing single-helix metamaterials at operation wavelengths of a few micrometers, but fabrication of $N=4$ helices in that range presently appears out of reach with regular DLW. However, recent progress regarding stimulated-emission-depletion (STED) DLW [18] raises hopes that even metallic $N=4$ helix metamaterials may become experimental reality in the not-too-distant future.

\section{Acknowledgments}

We thank Ralph von Baltz (KIT) for discussions. We acknowledge support by the Deutsche Forschungsgemeinschaft (DFG), the State of Baden-Württemberg, and the Karlsruhe Institute of Technology (KIT) through the DFG-Center for Functional Nanostructures (CFN) within subprojects A1.4 and A1.5. The project METAMAT is supported by the Bundesministerium für Bildung und Forschung (BMBF). The $\mathrm{PhD}$ education of $\mathrm{J}$. K. is embedded in the Karlsruhe School of Optics \& Photonics (KSOP). 